\section{Private collections hold back science}

Christian Foth and colleagues describe the spectacular eleventh specimen of the earliest bird, Archaeopteryx, including plumage features previously unknown for this pivotal taxon (Nature 511, 79-82; 2014). We are concerned, however, about access to this important specimen after its long-term loan to a public museum expires, when it will be returned to a private collection.

This Archaeopteryx has been registered under the 'Act to Prevent the Exodus of German Cultural Property' (see go.nature. com/xyk5lz), which requires its whereabouts to be recorded and prevents loss of German heritage. However, public access to such specimens remains at the owner's discretion.

Many journals refuse to publish characteristics of specimens held in private collections because the observations cannot be independently verified, which is counterproductive for the scientific community. Instead, journals should insist on a guarantee of future access as a condition of publication.

Paul M. Barrett, Martin C. Munt Natural History Museum, London, UK.

p.barrett@nhm.ac.uk

\section{Smart tools boost mental-health care}

Portable electronic devices such as smart phones and virtual-reality headsets provide clinicians with a powerful tool for improving evidence-based psychological treatments (see Nature 511, 287-289; 2014).

For example, they offer patients more-frequent access to therapy and are likely to boost compliance.

Internet-based cognitive behavioural therapy, for instance, is effective for conditions such as depression and anxiety. Immersive virtual reality helps for some phobias, including fear of flying and fear of heights, and holds promise for conditions such as eating disorders and post-traumatic stress disorder (see G. Riva CyberPsychol. Behav. 8, 220 230; 2005).

Smartphones, in conjunction with wearable sensors, convey information about patients' activity, location and even physiological responses, providing insight into how well they function in everyday life and guiding decisions on mental-health interventions.

Virtual reality could also be a useful tool for researchers investigating the neural basis of functional and dysfunctional psychological processes (see C. J. Bohil et al. Nature Rev. Neurosci. 12, 752-762; 2011). Andrea Gaggioli, Giuseppe Riva Catholic University; and Istituto Auxologico, Milan, Italy. andrea.gaggioli@unicatt.it

\section{Rules for assessing pain in lab animals}

New rules in the European Union directive for protecting laboratory animals call for a formal assessment of any pain that these animals might experience during scientific procedures (see go.nature.com/porg2x).

An upcoming workshop, organized by Germany's Federal Institute for Risk Assessment, will evaluate national guidelines on pain assessment drawn up in a similar workshop last year, with particular reference to 'harmful phenotypes' - animal lines that are likely to experience pain as a result of genetic alteration (see go.nature.com/8dhjcz).

Maintenance of such genetically altered animals, which carry the defining mutation at a single genetic locus, is currently subject to project authorization. This consent ensures that pain is monitored closely in these animals by veterinarians, animal-welfare officers, scientists and laboratory staff who are responsible for animal care. Breeding more animals for the purpose of assessing pain severity is not permitted, and neither is subjecting them to further pain - as inflicted, for example, by routine blood testing.

Participants in the next workshop, to be held in October, will collate experiences resulting from implementation of the 2013 guidelines and will develop a database for pain-assessment results from genetically altered animals.

Barbara Grune, Andreas

Hensel Federal Institute for

Risk Assessment (BfR), Berlin, Germany.

Gilbert Schönfelder BfR; and Charité-Universitätsmedizin Berlin, Germany. gilbert.schoenfelder@bfr.bund.de

\section{Early life is key to disease risk}

Lawrence O. Gostin calls for action to curb noncommunicable diseases (Nature 511, 147-149; 2014). Interventions in early life can also make a big difference.

There is now overwhelming evidence that factors in a child's early environment, during periods of developmental plasticity - including in utero - are major risk determinants for non-communicable disease in later life (see, for example, R. Barouki et al. Environ. Health 11, 42; 2012).

We should heed recommendations, based on findings in the field known as developmental origins of health and disease, that preventive strategies during fetal development and early childhood will ultimately be more effective in reducing the long-term burden of disease. (see, for example, S. C. Davies et al. Lancet 382, 1383-1384; 2013; and M. W. Gillman and D. S. Ludwig N. Engl. J. Med. 369, 2173-2175; 2013).

Jeffrey M. Craig Murdoch
Childrens Research Institute, The Royal Children's Hospital, Parkville, Australia. jeff.craig@mcri.edu.au Susan Prescott School of Paediatrics and Child Health, University of Western Australia, Perth, Australia.

\section{More inclusivity in PhD admissions}

More PhD programmes in science and engineering should adopt the mentoring model developed by Casey Miller and Keivan Stassun to boost numbers of female and minorities students (see Nature 510, 303-304; 2014). They should also use more-inclusive admissions criteria.

Efforts to democratize and diversify US higher education are placing a growing emphasis on applicants' social and economic origins (see, for example, W. G. Bowen et al. Equity and Excellence in American Higher Education Univ. Virginia Press, 2005). I suggest that admissions measures be expanded to include applicants who are firstgeneration college students, for example, or who were raised in poverty or in deprived singleparent families.

This broadened approach would fit with Miller and Stassun's recommendation that admissions practices be augmented with "proven markers of achievement, such as grit and diligence".

Kenneth Oldfield University of Illinois at Springfield, Illinois, USA.

koldf1@uis.edu

\section{CORRECTION}

In the Outlook article 'Funding by numbers' (Nature $\mathbf{5 1 1}$, S52-S53; 2014), the vertical axis of the graphic 'Scholarly spending' was wrongly labelled. It should have read 'Percentage of GDP spent on research and development'. 